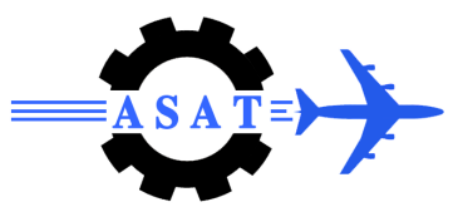

\title{
The Effect of Different Concentrations of $(R)-(+)$-WIN 55,212-2 on Wound Healing of Chondrocytes Monolayer.
}

\author{
A. Abdeldayem ${ }^{* \dagger}$, M. Yousseffi ${ }^{*}$, S.A. Khaghani ${ }^{* \dagger}$, M. Genedy ${ }^{* \dagger}$ and M.C.T. Denyer ${ }^{\dagger}$
}

\begin{abstract}
Number of studies has been conducted to highlight the anti-inflammatory and immunosuppressive properties of cannabinoids and also their potentials for prevention of cartilage degradation. Various wound healing techniques can be used to investigate the mechanisms of chondrocyte repair in monolayer or 3 dimensional tissue. Therefore, we investigated the effect of two different concentrations $(0.6$ and $0.9 \mu \mathrm{g} / \mathrm{ml})$ of the synthetic cannabinoid R (+) WIN55, 212-2 on the wound healing of chondrocyte monolayers using a simple scratch assay model. WIN55, 212-2 was found to increase both the migration and proliferation of chondrocytes cultured in a chondrogenic media, with increase in the wound closure rate. These findings highlight the potential use of the synthetic cannabinoid for improving the rate of wound closure as well as acting as an anti-inflammatory agent, which could be used to enhance tissue engineering protocols aimed at cartilage repair.
\end{abstract}

Keywords: WIN55, 212-2, chondrocyte, monolayer, cartilage, cell migration, cell proliferation.

\section{Introduction}

Cartilage is continually challenged during the process of endochondoral ossification by a low (as low as 1\%) oxygen micro-environment (Brittberg et al., 2001). This is mainly caused by very low (or no) blood supply to cartilage (Gowran et al., 2010, de Isla et al., 2010). The dramatic increase in the prevalence of cartilage disease and the poor ability of cartilage to repair (Slomianka, 2009) has increased the need for finding new ways for engineering cartilage. Chondrocytes play major role in maintaining cartilage homeostasis, where they maintain the balance between cartilage degradation and the biosynthesis as well as incorporation of matrix components (Steinmeyer and Daufeldt, 1997). Matrix metalloproteinases (MMPs) and other proteinases such as aggrecans and collagenases are mainly responsible for cartilage degradation, i.e. degrading main ECM components such as $\backslash$ collagen type II and proteoglycans (Cawston, 1998). Those proteinases are secreted by chondrocytes in a latent form, and inhibited by tissue inhibiters of metalloproteinases (TIMPs) (Steinmeyer et al., 1997). The ability of cartilage repair could therefore be increased if the secretion of TIMPs is increased or if the secretion of proteinases is reduced. Although cartilage may appear as a simple tissue containing one type of cell, the most suitable method for cartilage repair is natural regeneration or biological resurfacing space(Goldberg and Caplan, 1994) due to the complexity of its physiology.

\footnotetext{
* School of Engineering, Design \& Technology-Medical Engineering.

${ }^{\dagger}$ Institute of Pharmaceutical Innovation, University of Bradford, West Yorkshire, BD7 1DP, UK.
} 
Synthetic cannabinoids such as W Win-55,212-2 and HU-210 were found to affect the metabolism of chondrocytes reducing cartilage breakdown (Mbvundula et al., 2005 , Mbvundula et al., 2006). These cannabinoids were found to inhibit the production of IL-1 $\alpha$ induced nitric oxide (NO) (Mbvundula et al., 2005) thus reducing the degradation of cartilage due to MMPs. Ajulemic acid (AjA) another synthetic cannabinoid was found to suppress MMPs induced by TNF $\alpha$ or IL-1 $\alpha$ independently to Peroxisome proliferator-activated receptor gamma PPAR $\gamma$ (Ambrosio et al., 2007). It has also been found that these cannabinoids have an anti-inflammatory effect, which will prevent the degradation of cartilage in inflamed arthritic joints (McDougall et al., 2008 , Richardson et al., 2008). It is also known that cannabinoid system is present on the ancillary tissue and cells in cartilage as well as fibroblasts, myofibroblasts, MSCs and synoviocytes (Richardson et al., 2008 , McPartland, 2008 , Idris et al., 2009). Another observation showing the importance of the cannabinoid system in maintenance of the homeostasis of joints infected with OA an RA was the up regulation of the cannabinoid system in synoviol fluid and tissue sampled from patients with OA and RA (Richardson et al., 2008).

Although extensive studies have been conducted on the role of cannabinoids in bone metabolism (Idris and Ralston, 2010), so far, the effect of cannabinoid on chondrocyte wound repair has not been investigated and its repair mechanism has not been fully understood. The aim of this work was to investigate the effect of WIN55, 212-2 on wound healing of chondrocytes monolayer.

\section{Materials and Methods}

\section{Materials}

The following reagents were purchased and used in this work:

WIN55, 212-2, Trypsin, Collagenase, Hank's based salt solution (HBSS), L-glutamine, Ascorbic acid, penicillin streptomycin, Fungizone, and Fetal bovine serum (FBS) (Sigma Aldrich, UK). A mixture of High glucose $(4500 \mathrm{mg} / 1$ glucose) Dulbecco's modified eagle medium and Ham's F12 (DMEM/Ham's F12, 1:1) without L-Glutamine (PromoCell, UK). Ethanol and distilled water (University of Bradford, UK).

\section{Chondrocyte Cell Culture and Isolation}

Sprague-Dawley rats (6-3 days old) in accordance with EU guidelines (86/609/EEC). After removing the skin from the isolated legs and washing them in HBSS and removing the muscles and tendons around the knee joint using scalpel and forceps, the patella and the patella tendons were removed to reduce the risk of having tenocytes in the culture. The epiphyses was then isolated from both the femur and tibia. The epiphyses was washed in $70 \%$ ethanol (diluted with distilled water) to reduce the risk of bacterial contamination.

Chondrocytes were isolated from the epiphyses by using enzymatic digestion. The epiphyses was first immersed in $15 \mathrm{ml}$ Trypsin at $37^{\circ} \mathrm{C}$, the combination was stirred using a magnetic stirrer for 15 minutes. After 15 minutes the supernatant was removed and mixed in $50 \mathrm{ml}$ sterile tube with $4 \mathrm{ml}$ of isolation media (DMEM/Hams'F12 supplemented with $10 \% \mathrm{FBS}, 1 \%$ L-Glutamine,1\% Ascorbic acid, 2\% penicillin-streptomycin and 1\% Fungizone). This procedure was repeated 3 times.

The remaining tissue was then immersed in $2 \mathrm{ml}$ collagenase at $37^{\circ} \mathrm{C}$ for crude enzymatic digestion and stirred using a magnetic stirrer for 1 hour. After this the supernatant was 
removed and placed with the cells isolated from the previous enzymatic digestion in a $15 \mathrm{ml}$ sterile centrifuge tube and centrifuged at 1300rpm at room temperature for 5 minutes. The isolation media was removed from the centrifuge tube and $6 \mathrm{ml}$ of culture media (DMEM/Hams'F12 supplemented with 10\% FBS, 1\% L-Glutamine, 1\%Ascorbic acid, 1\% penicillin-streptomycin and $0.2 \%$ Fungizone) was added to the chondrocytes pellet. The chondrocytes suspension was passed through a $40 \mu \mathrm{m}$ nylon mesh into a sterile petri dish to reduce the risk of contamination due to the presence of tissue debris in the culture media.

The supernatant was split between two T75 culture flasks and $12 \mathrm{ml}$ of culture media was added to each flask and incubated in a humidified atmosphere $\left(95 \%\right.$ air and $\left.5 \% \mathrm{CO}_{2}\right)$ at $37^{\circ} \mathrm{C}$. The culture media was replaced after 24 hours to remove the non-adherent cells. Cells were passaged upon reaching $80-90 \%$ confluency to a maximum of 3 passages, where the medium was replaced every 3-4 days.

\section{Preparation of the WIN55, 212-2 Solution}

WIN55, 212-2 was dissolved in ethanol to produce two concentrations. The stock solution was prepared by dissolving $6 \mathrm{mg}$ and $9 \mathrm{mg}$ of WIN55, 212-2 in $1 \mathrm{ml}$ ethanol to produce a concentration of $6 \mathrm{mg} / \mathrm{ml}$ and $9 \mathrm{mg} / \mathrm{ml}$ respectively. The WIN55, 212-2 was then stored at $4^{\circ} \mathrm{C}$ as advised by the supplier for later use. $0.1 \mathrm{ml}$ of each of the stock solutions was added to $1 \mathrm{ml}$ of ethanol to reach a final concentration of $0.6 \mathrm{mg} / \mathrm{ml}$ and $0.9 \mathrm{mg} / \mathrm{ml}$.

\section{Scratch Assay Technique}

Chondrocytes from the 3rd passage were cultured to full confluency in four T25 culture flasks. Each flask was scratched using the tip of a $1 \mathrm{ml}$ sterile transfer pipette, to reach a wound width of $\sim 320 \mu \mathrm{m} \pm 30 \mu \mathrm{m}$. New culture media $(5 \mathrm{ml})$ was added to the flasks and then $5 \mu \mathrm{m}$ of ethanol was also added to be used as a negative control. $5 \mu 1$ of the final solutions of WIN55, 212-2 $(0.6 \mathrm{mg} / \mathrm{ml}$ and $0.9 \mathrm{mg} / \mathrm{ml})$ was added to two of the flasks to dilute WIN55, 212-2 with final concentrations of $0.6 \mu \mathrm{g} / \mathrm{ml}$ and $0.9 \mu \mathrm{g} / \mathrm{ml}$ in the culture media. The 4 th flask had only culture media to be used as control. Each scratch was monitored and imaged at 3 different regions every 2 hours over a period of 18 hours by a phase contrast microscope at x10 magnification.

Image J software was used to image and measure the width of the scratches at different regions. For each region 10 readings were recorded and the means of those readings were calculated. The means of the readings for 3 regions were averaged for each case and then used to determine the rate of wound closure for all 4 different culture flasks.

\section{Proliferation Assay}

The effect of WIN55, 212-2 on the proliferation rate of chondrocytes was assessed by seeding chondrocytes in two T25 culture flasks at an initial density of $13 \times 10_{4}$ cells $/ \mathrm{ml}$, i.e. $65 \times 104$ cells in T25 culture flask. WIN55, 212-2 was added to one of the flasks with a concentration of $0.9 \mu \mathrm{g} / \mathrm{ml}$ and the other flask had only culture media used as control. The cells were incubated in a humidified atmosphere $\left(95 \%\right.$ air and $\left.5 \% \mathrm{CO}_{2}\right)$ at $37^{\circ} \mathrm{C}$ for 72 hours. After 72 hours the cells were washed two times with HBSS then $1 \mathrm{ml}$ of Trypsin was added to each culture flask and the cells were incubated in humidified atmosphere $\left(95 \%\right.$ air and $\left.5 \% \mathrm{CO}_{2}\right)$ at $37^{\circ} \mathrm{C}$ for 3 minutes. The cells were monitored under the phase contrast microscope at x10 magnification to make sure that all the cells had detached. After this $1 \mathrm{ml}$ of culture media was added to each flask to stop the effect of trypsin. Cell count was preformed five times for each flask using haemocytometer and the average of the 5 readings was calculated. This experiment was repeated 3 times to validate the results obtained. 


\section{Results and Discussion}

\section{Effects of WIN55, 212-2 on the Rate of Wound Closure}

The exposure of the wounded chondrocyte monolayer to WIN55, 212-2 lead to an increase in the rate of wound closure. The rate of wound closure was increased by increasing the concentration of WIN55, 212-2 in the culture media. As shown in Figures 1 and 2, the chondrocytes treated with $0.9 \mu \mathrm{g} / \mathrm{ml}$ of WIN55, 212-2 were the first to heal at less than 12 hours with healing rate of $9.27 \% / \mathrm{h}$. The wounded chondrocyte monolayer treated with $0.6 \mu \mathrm{g} / \mathrm{ml}$ of WIN55, 212-2 healed at $14 \mathrm{~h}$ with healing rate of $8.23 \% / \mathrm{h}$. The healing rate of the chondrocytes treated with ethanol only $(=7.15 \% / \mathrm{h})$ was lower than the rate of the chondrocytes treated with $0.6 \mu \mathrm{g} / \mathrm{ml}$ of WIN55, 212-2, where the wound closed after $\sim 16$ hours. The rate of wound healing for the untreated chondrocytes control was much lower $(6 \% / \mathrm{h})$ than the rate of the chondrocytes treated with higher concentration, where the wound was not completely healed after 18 hours.

\section{Proliferation Assay}

The average number of chondrocytes treated with $0.9 \mu \mathrm{g} / \mathrm{ml}$ WIN55, 212-2 counted after 72 hours in T25 culture flask, was $189 \times 104$ cells, which are 2.9 times higher than the number of cells $(65 \times 104$ cells $)$ initially seeded. On the other hand, the average number of chondrocytes not treated (control) was $136 \times 104$ cells, which are 2.09 times the number of cells initially seeded. This clearly shows that treating chondrocytes with WIN55, 212-2 at a concentration of $0.9 \mu \mathrm{g} / \mathrm{ml}$ increases the rate of proliferation.

\section{Discussion}

The mechanically scratched monolayers had damaged/injured chondrocytes in all four different flasks. Damaged chondrocytes release nitric oxide (NO) (Im and Shin, 2002) which is a highly reactive free radical that has been implicated in tissue injury in OA (Dinarello, 1996). NO has also been known to exert detrimental effects on chondrocyte function, causing inhibition of collagen and procollagen synthesis (Selvi et al., 2008). The prevention of chondrocytes to secrete ECM components such as collagen and proteoglycan will reduce the rate of cell migration and cell proliferation thus reducing the rate of wound closure. As mentioned earlier, WIN55, 212-2 has been found to reduce the IL-1 $\alpha$ induced nitric oxide (NO) (Mbvundula et al., 2005) thus reducing the degradation of cartilage due to MMPs. This explains the increase in the rate of wound closure obtained in the sample treated with $0.9 \mu \mathrm{g} / \mathrm{ml}$ WIN55, 212-2, which is due to an increase in both cell migration and proliferation.

The increase in the rate of wound closure also suggests that WIN55, 212-2 has an up regulation effect on the metabolic activity of chondrocytes. This can be observed by both the increase in cell migration and proliferation rate. The increase in proliferation rate can also be reasoned by the down regulation of $\mathrm{NO}$, where it was found to cause apoptosis by inhibition of $\beta$-1 integrin-dependent adhesion to ECM (Mbvundula et al., 2006, Hood JD, 2002). 


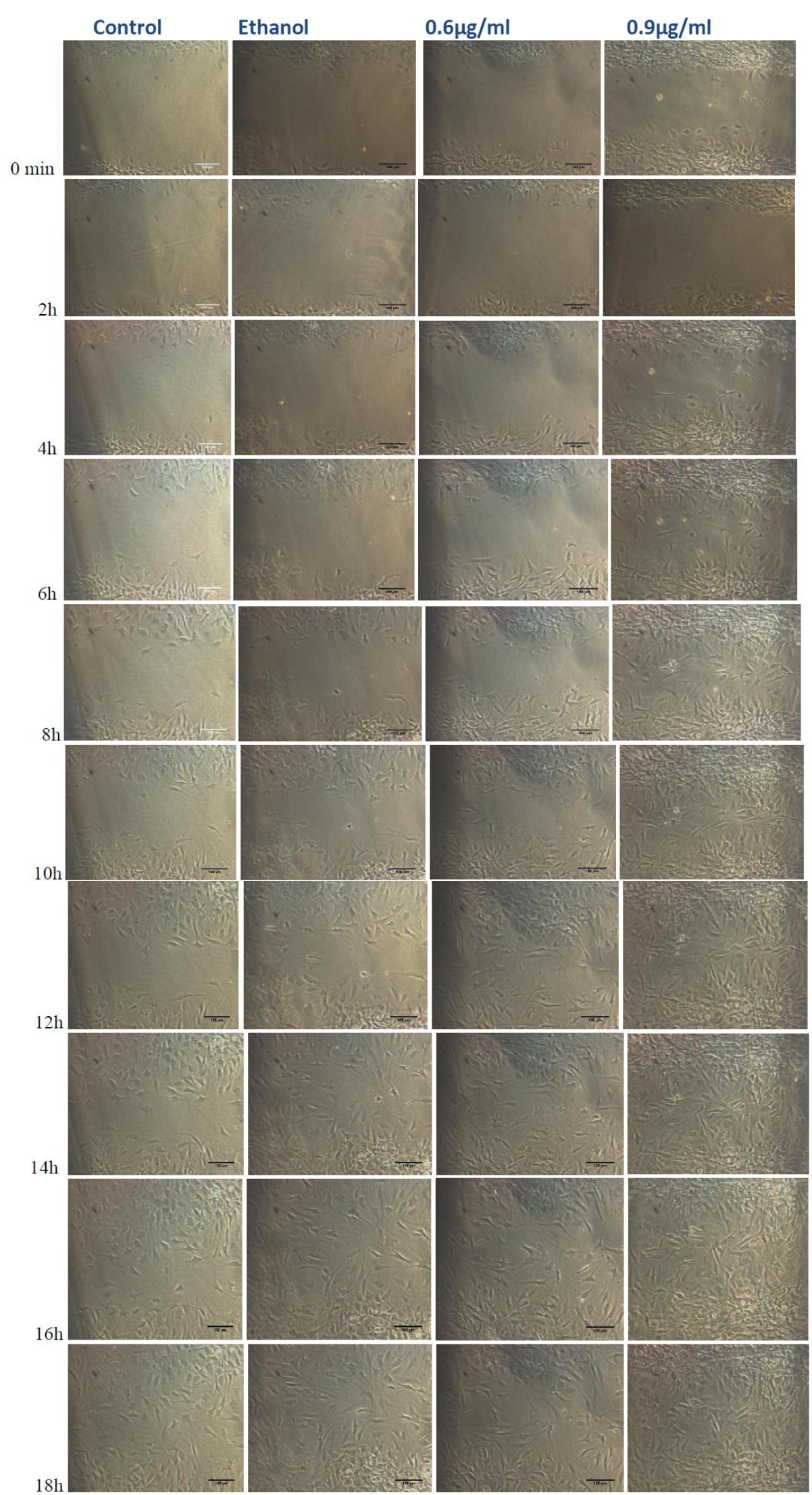

Figure 1 Images of the wound healing for the chondrocyte monolayer cultured in monolayer, for control, ethanol, $0.6 \mu \mathrm{g} / \mathrm{ml}$ and $0.9 \mu \mathrm{g} / \mathrm{ml}$ WIN55, 212-2 for a period of 16hours (Scale bar $=100 \mathrm{~m}$ ). 


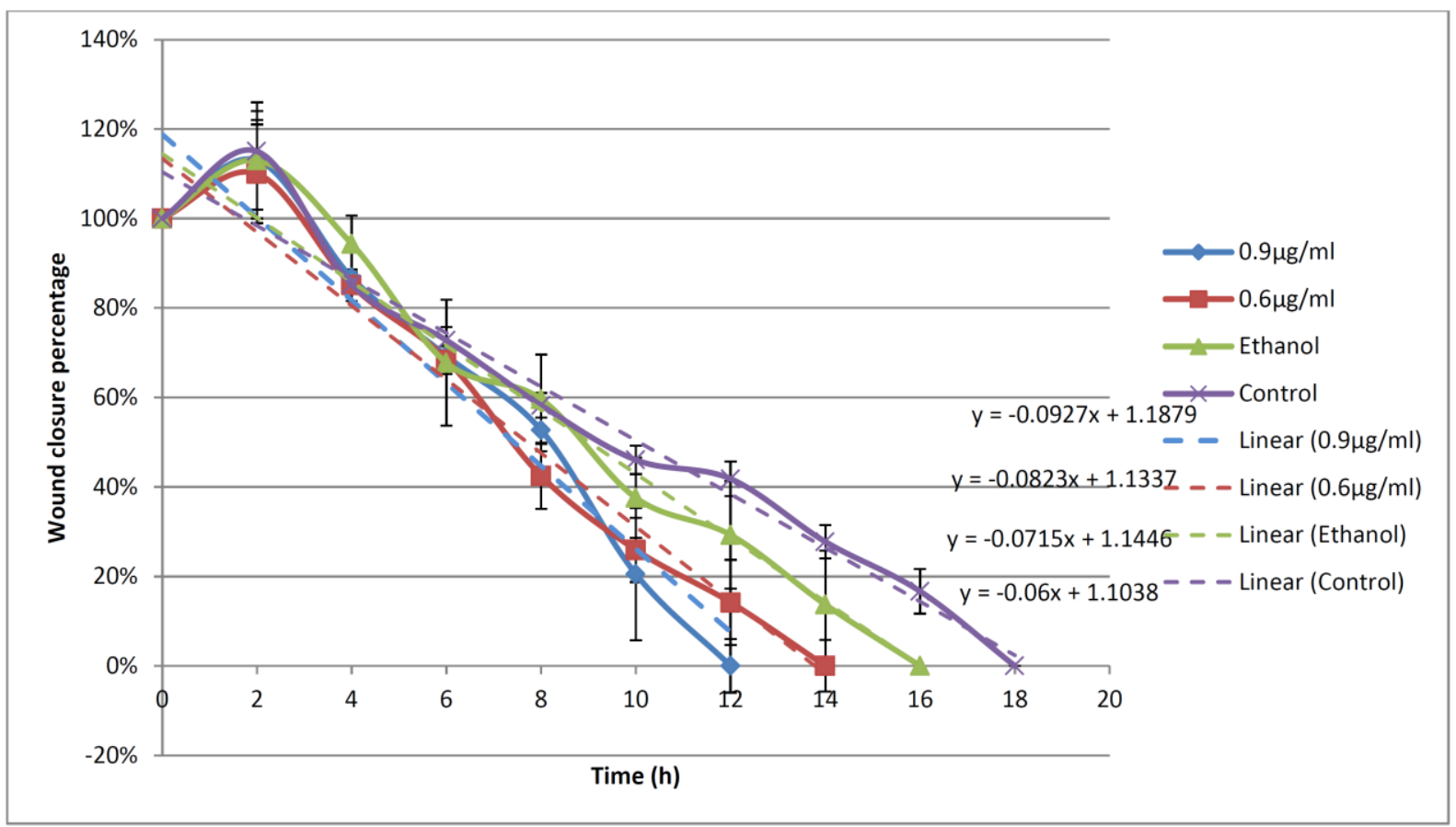

Figure 2 Rate of chondrocytes wound closure for control, ethanol, $0.6 \mu \mathrm{g} / \mathrm{ml}$ WIN55, 212-2 and $0.9 \mu \mathrm{g} / \mathrm{ml}$ WIN55, 212-2 for a period of 18hours

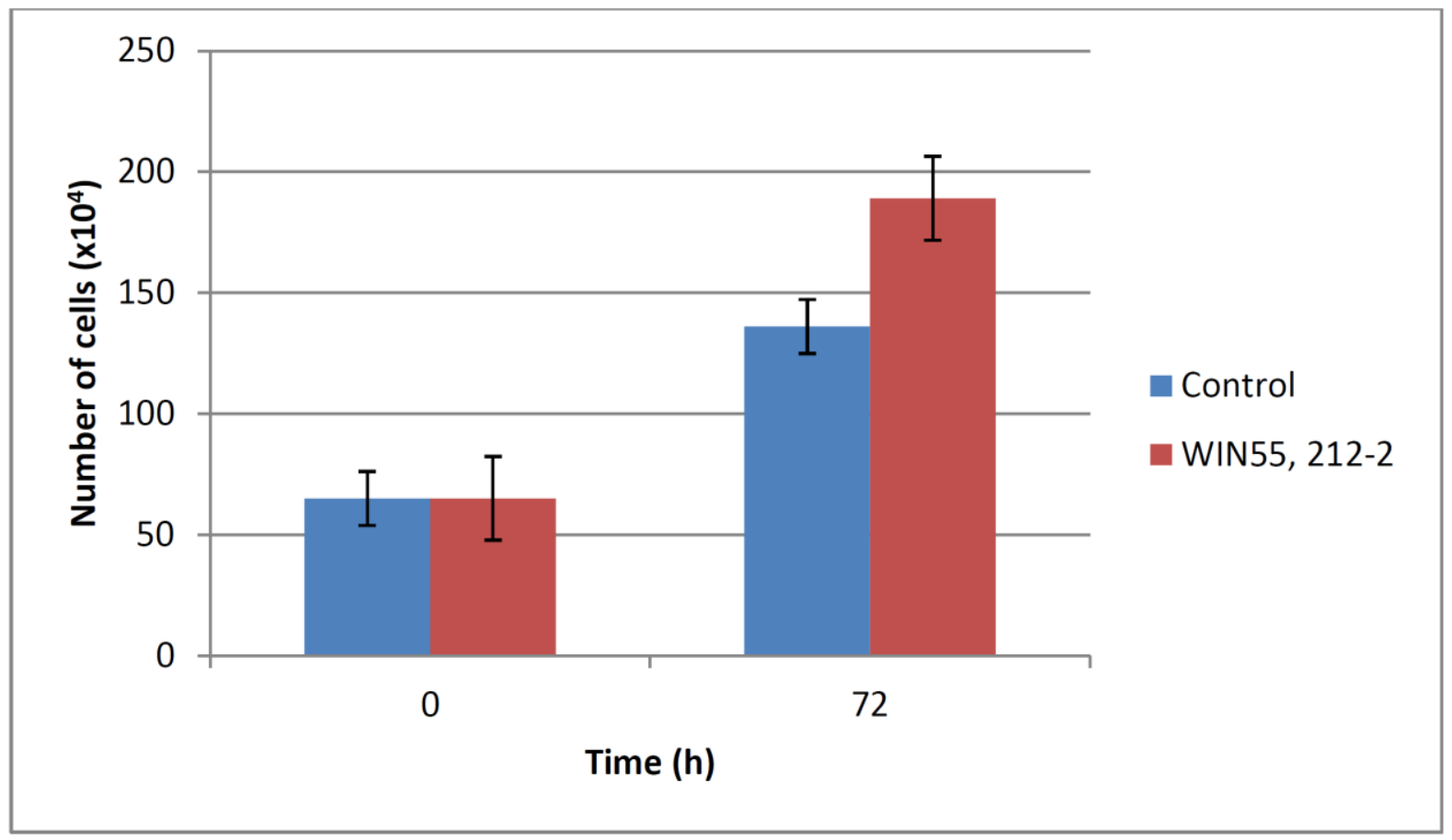

Figure 3 Proliferation of the untreated chondrocytes and the chondrocytes treated with $0.9 \mu \mathrm{g} / \mathrm{ml}$ WIN55,212-2. 


\section{Conclusions}

The synthetic cannabinoid WIN55, 212-2 increased the rate of wound closure from $6 \% / \mathrm{h}$ (for control) to $9.27 \% / \mathrm{h}$ (for $0.9 \mu \mathrm{g} / \mathrm{ml}$ WIN55, 212-2) by increasing both cell migration and proliferation. Previous studies had shown the anti-inflammatory and immunosuppressive effects of WIN55, 212-2. Further investigations are needed to quantify the effects of WIN55, 212-2 on the mechanisms of chondrocytes wound closure via immunostaining. Our preliminary results therefore show promising wound healing properties of the synthetic cannabinoid using a simple wound assay technique.

\section{References}

[1] AMBROSIO, A. L., et al. (2007) Ajulemic acid, a synthetic nonpsychoactive cannabinoid acid, bound to the ligand binding domain of the human peroxisome proliferator-activated receptor gamma. In: J Biol Chem. Vol. 282. United States: pp. 18625-33.

[2] BRITTBERG, M., et al. (2001) Autologous chondrocytes used for articular cartilage repair: an update. Clin Orthop Relat Res, (391 Suppl), S337-48.

[3] CAWSTON, T. (1998) Matrix metalloproteinases and TIMPs: properties and implications for the rheumatic diseases. In: Mol Med Today. Vol. 4. England: pp. 130-7.

[4] DE ISLA, N., et al. (2010) Introduction to tissue engineering and application for cartilage engineering. Biomed Mater Eng, 20 (3), 127-33.

[5] DINARELLO, C. A. (1996) Biologic basis for interleukin-1 in disease. Blood, 87 (6), 2095-147.

[6] GOLDBERG, V. M. and CAPLAN, A. I. (1994) Biological resurfacing: an alternative to total joint arthroplasty. Orthopedics, 17 (9), 819-21.

[7] GOWRAN, A., et al. (2010) Tissue Engineering of Cartilage; Can Cannabinoids Help? Pharmaceuticals, 3.

[8] HOOD JD, C. D. (2002) Role of integrins in cell invasion and migration. [Nat Rev Cancer. 2002] - PubMed result. MEDLINE.

[9] IDRIS, A. and RALSTON, S. (2010) Cannabinoids and bone: friend or foe? Calcif Tissue Int, 87 (4), 285-97.

[10] IDRIS, A. I., et al. (2009) Cannabinoid receptor type 1 protects against age-related osteoporosis by regulating osteoblast and adipocyte differentiation in marrow stromal cells. In: Cell Metab. Vol. 10. United States: pp. 139-47.

[11] IM, G. I. and SHIN, S. R. (2002) Changes in the production and the effect of nitric oxide with aging in articular cartilage: an experimental study in rabbits. Acta Orthop Scand, 73 (1), 6-10.

[12] MBVUNDULA, E. C., et al. (2005) Effects of cannabinoids on nitric oxide production by chondrocytes and proteoglycan degradation in cartilage. In: Biochem Pharmacol. Vol. 69. England: pp. 635-40.

[13] MBVUNDULA, E. C., et al. (2006) Arthritis and cannabinoids: HU-210 and Win55,212-2 prevent IL-1alpha-induced matrix degradation in bovine articular chondrocytes in-vitro. J Pharm Pharmacol, 58 (3), 351-8.

[14] MCDOUGALL, J., et al. (2008) In vivo effects of CB2 receptor-selective cannabinoids on the vasculature of normal and arthritic rat knee joints. Br J Pharmacol, 153 (2), 35866.

[15] MCPARTLAND, J. M. (2008) Expression of the endocannabinoid system in fibroblasts and myofascial tissues. In: J Bodyw Mov Ther. Vol. 12. United States: pp. 169-82. 
[16] RICHARDSON, D., et al. (2008) Characterisation of the cannabinoid receptor system in synovial tissue and fluid in patients with osteoarthritis and rheumatoid arthritis. Arthritis Res Ther, 10 (2), R43.

[17] SELVI, E., et al. (2008) Inhibitory effect of synthetic cannabinoids on cytokine production in rheumatoid fibroblast-like synoviocytes. Clinical and Experimental Rheumatology, 26 (4), 574-581.

[18] SLOMIANKA, L. (2009) Blue Histology - Skeletal Tissues - Cartilage. School of anatomy and human biology- The University of Western Australia, Available.

[19] STEINMEYER, J. and DAUFELDT, S. (1997) Pharmacological influence of antirheumatic drugs on proteoglycans from interleukin-1 treated articular cartilage. Biochem Pharmacol, 53 (11), 1627-35.

[20] STEINMEYER, J., et al. (1997) The proteoglycan metabolism, morphology and viability of articular cartilage treated with a synthetic matrix metalloproteinase inhibitor. Res Exp Med (Berl), 197 (2), 63-79. 